\title{
Jawarneh Manifold with Semi Symmetric Metric Connection
}

\author{
Musa A.A. Jawarneh \\ University of Jeddah, Al-Kamil Faculty of Science and Arts, Department of mathematics, P.O.Box1 10, Alkamil
} 21931, Al-Kamil, Saudi Arabia.

\begin{abstract}
Jawarneh manifold along with semi-symmetric metric connectionhave been studied, and some of its geometric properties are derived, and some application in general relativity are mentioned.Also a new example of Jawarneh manifold is given.
\end{abstract}

Keywords: Riemannian manifold, semi-symmetric metric connection,locally flat manifold, locally conharmonically flatmanifold, conformally flatmanifold, Jawarneh manifold, general relativity.

Mathematics Subject Classification (2010):53C55(primary); 58C25(secondary).

\section{Introduction}

In a recent paper [1] the author introduced a new type of Riemannian manifold called Jawarneh manifold, i. e., a Riemannian manifold $\left(\mathrm{M}^{\mathrm{n}}, \mathrm{g}\right)(\mathrm{n} \geq 2)$, such that its curvature tensor $\mathrm{R}$ satisfies the relation,

1.1) $\mathrm{R}(\mathrm{X}, \mathrm{Y}, \mathrm{Z})=\mathrm{k}[\mathrm{S}(\mathrm{Y}, \mathrm{Z}) \mathrm{X}-\mathrm{S}(\mathrm{X}, \mathrm{Z}) \mathrm{Y}]$,

wherek is constant and $\mathrm{Q}$ is the symmetric endomorphism of the Ricci tensor $\mathrm{S}$ such that,

1.1) $\mathrm{S}(\mathrm{X}, \mathrm{Y})=\mathrm{g}(\mathrm{QX}, \mathrm{Y})$.

A linear connection $\widetilde{\nabla}$ on n-dimensional Riemannian manifold $\left(M^{n}, g\right)$ is called semi-symmetric connection [3] if the torsion tensor $\mathrm{T}$ of the connection satisfies,

1.3) $\mathrm{T}(\mathrm{X}, \mathrm{Y})=\alpha(Y) X-\alpha(X) Y$,

For every vector fields $\mathrm{X}, \mathrm{Y}$ on $\mathrm{M}$, and $\alpha$ is a 1 -form associated with the torsion tensor $\mathrm{T}$ of the connection $\widetilde{\nabla}$ given by,

1.4) $\alpha(X)=g(X, \rho)$,

where $\rho$ is a vector field associated with the 1 -form $\alpha$. The 1 -form $\alpha$ is called the associated 1 -form of the semisymmetric connection $\widetilde{\nabla}$ and the vector field $\rho$ is called the associated vector field of the semi-symmetric connection $\widetilde{\nabla}$. A semi-symmetricconnection $\widetilde{\nabla}$ is called a semi-symmetric metric connection [3] if it satisfies also,

1.5) $\widetilde{\nabla} \mathrm{g}=0$.

The relation between the Riemannian connection and the semi-symmetric metric connection is given by [3],

1.6) $\widetilde{\nabla}_{X} Y=\nabla_{X} Y+\alpha(Y) X-g(X, Y) \rho$.

The covariant differentiation of a 1 -form $\omega$ with respect to $\widetilde{\nabla}$ is given by [3]

1.7) $\left(\widetilde{\nabla}_{X} \omega\right)(Y)=\left(\nabla_{X} \omega\right)(Y)+\omega(X) \alpha(Y)-\omega(\rho) g(X, Y)$.

Now if $\widetilde{R}$ and $\mathrm{R}$ denote respectively the curvature tensors of $\nabla$ and $\widetilde{\nabla}$ then [3]

1.8) $\tilde{R}(X, Y) Z=R(X, Y) Z-H(Y, Z) X+H(X, Z) Y-g(Y, Z) L X+g(X, Z) L Y$

where $\mathrm{H}$ is a tensor field of type $(0,2)$ given as

1.9) $H(X, Y)=g(L X, Y)=\left(\nabla_{X} \alpha\right)(Y)-\alpha(X) \alpha(Y)+\frac{1}{2} \alpha(\rho) g(X, Y)$,

For any vector fields $X, Y$.

Let $X, Y \in T_{p}(M)$ at a point $p \in M$. Let $\gamma$ be a plane spanned by $X, Y$. Thenthe sectional curvature with respect to the section $\gamma$ is defined by [2],

1.10) $k(\gamma)=-\frac{R(X, Y, X, Y)}{g(X, X) g(Y, Y)-g(X, Y)^{2}}$.

Sectional curvature $\mathrm{k}(\gamma)$ is uniquely determined by the section $\gamma$ and isindependent of the vectors $\mathrm{X}, \mathrm{Y}$ in the section. If the sectional curvature $\mathrm{k}(\gamma)$ is a constant for allsections $\gamma$ at each point of $\mathrm{M}$, then $\mathrm{M}$ is said to be a spaceof constant curvature and we have [2],

1.11) $\mathrm{R}(\mathrm{X}, \mathrm{Y}) \mathrm{Z}=\mathrm{h}[\mathrm{g}(\mathrm{Y}, \mathrm{Z}) \mathrm{X}-\mathrm{g}(\mathrm{X}, \mathrm{Z}) \mathrm{Y}]$,

for any $\mathrm{C}^{\infty}$ vector fields $\mathrm{X}, \mathrm{Y}, \mathrm{Z}$ on $\mathrm{M}$ where $\mathrm{h}$ is a constant.

The conformal curvature tensorC and the projective curvature tensor $\mathrm{P}$ of a Riemannian manifold[2]are defined as,

$$
\begin{aligned}
& \text { 1.12) } C(X, Y, Z)= \\
& R(X, Y, Z)-\frac{1}{(n-2)}[S(Y, Z) X-S(X, Z) Y+g(Y, Z) Q X-g(X, Z) Q Y]+\frac{r}{(n-1)(n-2)}[\mathrm{g}(\mathrm{Y}, \mathrm{Z}) \mathrm{X}-\mathrm{g}(\mathrm{X}, \mathrm{Z}) \mathrm{Y}], \\
& \text { 1.13) } P(X, Y, Z)=R(X, Y, Z)-\frac{1}{(n-1)}[S(Y, Z) X-S(X, Z) Y] .
\end{aligned}
$$


A Riemannian manifold is said to be locally Projactivelyflat if,

1.14) $P(X, Y, Z)=0$.

Yano [3] proved the following important existence theorem,

Theorem1.1) In order that a Riemannian manifold admits a semi-symmetric metric connection whose curvature tensor vanishes, it is necessary and sufficient that the Riemannian manifold is conformally flat.

Also it is proved in [4] that,

Theorem1.2) A Riemannian manifold of constant curvature is conformally flat.

The Ricci tensor $\tilde{S}$ and the scalar curvature $\tilde{r}$ of Jawarneh manifold along with a semi-symmetric metric connection $\widetilde{\nabla}$ is defined as,

1.16) $\tilde{S}(Y, Z)=\left(C_{1}^{1} \tilde{R}\right)(X, Y)=\sum_{i=1}^{n} \tilde{R}\left(E_{i}, Y, Z, E_{i}\right)$,

1.17) $\tilde{r}=\sum_{i=1}^{n} \tilde{S}\left(E_{i}, E_{i}\right)$,

where $E_{i}, \mathrm{i}=1, \ldots, \mathrm{n}$ are orthonormal vector fields on $\mathrm{M}$.

In section 2 it is been shown that if Jawarnehmanifold admit a semi-symmetric metric connection then it is locally flat manifold if and only if its scalar curvature vanishes. Also it is tensor $\mathrm{H}$ defined by (1.9) is symmetric, and the 1 -form $\alpha$ is closed.Further it is shown that the Riemannian curvature tensor is equivalent to the conformal curvature tensor. This result bears some geometric characteristic of the manifold.Also we got the eigen value of the tensor $\mathrm{H}$ and the eigen vector of the eigen value.Finally we proved thatJawarnehmanifold admit a semi-symmetric metric connection and obtained some properties of the projective curvature tensor.

Section 3 was devoted for a new example of Jawarneh manifold.

Contracting (1.8) we get,

\section{2- Semi-symmetric Metric Connection}

2.1) $\tilde{S}(Y, Z)=S(Y, Z)-(n-2) H(Y, Z)-\operatorname{ag}(Y, Z)$,

where a denote the trace of H.From which we have,

2.2) $\tilde{Q}(X)=Q(X)-(n-2) L(X)-a(X)$.

Further contraction yields,

2.3) $\tilde{r}=r-2(n-1) a$.

Now let us define A Riemannian manifold $\left(\mathrm{M}^{\mathrm{n}}, \mathrm{g}\right)(\mathrm{n}>2)$ as a Jawarneh manifold admitting a semi-symmetric metric connection $\widetilde{\nabla}$ such that its curvature tensor $\widetilde{R}$ satisfy the relation,

2.4) $\widetilde{R}(\mathrm{X}, \mathrm{Y}, \mathrm{Z})=k[\widetilde{\mathrm{S}}(\mathrm{Y}, \mathrm{Z}) \mathrm{X}-\widetilde{\mathrm{S}}(\mathrm{X}, \mathrm{Z}) \mathrm{Y}]$,

and denote such a manifold by $[\mathrm{J}, \widetilde{\nabla}]$.

Contracting (2.4) we get,

2.5) $\tilde{S}(X, W)=\frac{k \tilde{r}}{(1+k)} g(X, W)$.

Further contraction and since on Jawarneh manifold $\mathrm{k}=\frac{1}{n-1}$ we have,

2.6) $\tilde{r}=0$.

Thus we can state,

Theorem 2.1) $[\mathrm{J}, \widetilde{\nabla}]$ is locally flat manifold.

From (2.5), (2.6) and (2.1) we can have,

2.7) $\mathrm{H}(\mathrm{X}, \mathrm{Y})=\mathrm{H}(\mathrm{Y}, \mathrm{X})$.

Thus we can state,

Theorem2.2) On $[\mathrm{J}, \widetilde{\nabla}]$ the tensor H defined by (1.9) is symmetric.

Using (1.9) on (2.7) we can get,

$2.8)\left(\nabla_{X} \alpha\right)(Y)-\left(\nabla_{Y} \alpha\right)(X)=0$.

Thus we can state,

Theorem2.3) On $[\mathrm{J}, \widetilde{\nabla}]$ the 1 -form $\alpha$ is closed.

From (1.8) by changing $X, Y$, and $Z$ cyclically and by virtue of (2.7) we can have,

2.9) $\tilde{R}(X, Y) Z+\tilde{R}(Y . Z) X+\tilde{R}(Z, X) Y=0$,

2.10) $\tilde{R}(X, Y, Z, W)=\tilde{R}(Z, W, X, Y)$.

Thus we can state,

Theorem2.4) On $[\mathrm{J}, \widetilde{\nabla}]$ we have equations (2.9) and (2.10).

Now from (2.5), (2.6) and (2.1) we have,

2.11) $S(Y, Z)=(n-2) H(Y, Z)+a g(Y, Z)$.

Contracting we get,

2.12) $a=\frac{r}{2(n-1)}$. 
Therefore (2.11) will reduce to the form,

2.13) $H(Y, Z)=\frac{1}{(n-2)} S(Y, Z)-\frac{r}{2(n-1)(n-2)} g(Y, Z)$, or,

2.14) $L X=\frac{1}{(n-2)} Q X-\frac{r X}{2(n-1)(n-2)}$.

Using (2.12) and (2.14) on (1.8) we get,

2.15) $\tilde{R}(X, Y) Z=$

$R(X, Y) Z-\frac{1}{(n-2)}[S(Y, Z) X-S(X, Z) Y]-\frac{1}{(n-2)}[g(Y, Z) Q X-g(X, Z) Q Y]+\frac{r}{(n-1)(n-2)}[g(X, Z) Y-$ $g(Y, Z X]$.

This can be written by virtue of (1.12) as,

2.16) $\tilde{R}(X, Y) Z=C(X, Y, Z)$.

Thus we can state,

Theorem2.5)On [J, $\widetilde{\nabla}]$ the Riemannian curvature tensor is equivalent to the conformal curvature tensor.

Also in consequence of theorem (2.1), theorem (1.2) and since Jawarneh manifold is of constant curvature we can state,

Theorem2.6) $[\mathrm{J}, \widetilde{\nabla}]$ is conformallyflat manifold.

Hence in $[J, \widetilde{\nabla}]$ for dimensions 2 and 3 the curvature tensor vanishes identically, whilein dimensions $\geq$ 4 , the curvature is generally nonzero. If the curvature tensor vanishes in dimensions $\geq 4$, then the metric is locally conformally flat: there exists a local coordinate system in which the metric tensor is proportional to a constant tensor. This fact was a key component of Nordstrom's theory of gravitation, which was a precursor of general relativity.

Further in $[\mathrm{J}, \widetilde{\nabla}]$ the curvature tensor is the only part of the curvature that exists in free space (a solution of the vacuum Einstein equation) and it governs the propagation of gravitational radiation through regions of space devoid of matter. Also in $[\mathrm{J}, \widetilde{\nabla}]$ the curvature tensor is a measure of the curvature of space-time or, more generally, a pseudo-Riemannian manifold. Like the Riemann curvature tensor, curvature tensor in $[\mathrm{J}, \widetilde{\nabla}]$ expresses the tidal force that a body feels when moving along a geodesic, but only how the shape of the body is distorted by the tidal force.

Now we have from (2.8) and (1.9) that,

2.17) $H(X, Y)=\frac{1}{2} \alpha(\rho) g(X, Y)$.

Thus we can state,

Theorem2.7) On $[\mathrm{J}, \widetilde{\nabla}] \frac{1}{2} \alpha(\rho)$ is an Eigen value of the tensor $\mathrm{H}$ defined by (1.9) with $\rho$ is an Eigen vector of the Eigen value.

Using (2.17), (2.11) and (2.12) on (2.15) we can get,

2.18) $\tilde{R}(X, Y) Z=R(X, Y, Z)-\left[\frac{\alpha(\rho)}{2}+\frac{r}{(n-1)(n-2)}\right][g(Y, Z) X-g(X, Z) Y]-[g(Y, Z) L X-g(X, Z) L Y]$.

In a consequence of theorem (2.6) and theorem (1.1) we can state,

Theorem2.8)Jawarneh manifold admits a semi-symmetric metric connection.

The projective curvature tensor of $[\mathrm{J}, \widetilde{\nabla}]$ can be defined as,

2.19) $\tilde{P}(X, Y, Z)=\tilde{R}(X, Y, Z)-\frac{1}{(n-1)}[\tilde{S}(Y, Z) X-\tilde{S}(X, Z) Y]$.

Thus by virtue of (2.5), (2.6) and theorem (2.1) we can stste,

Theorem2.9) $[\mathrm{J}, \widetilde{\nabla}]$ is locally projectivelyflat manifold.

In consequence of (2.5), (2.6), (2.8), (2.9), (2.10) and (2.19) we can easily prove,

Theorem2.10) On $[\mathrm{J}, \widetilde{\nabla}]$ we have:

1) $\tilde{P}(X, Y) Z+\tilde{P}(Y, Z) X+\widetilde{P}(Z, X) Y=0$,

2) $\tilde{P}(X, Y) Z=\tilde{R}(X, Y) Z$,

3) $\tilde{P}(X, Y, Z, W)=\tilde{P}(Z, W, X, Y)$,

4) $C_{1}^{1} \tilde{P}(X, Y) Z=C_{3}^{1} \tilde{P}(Y, Z) X=0$.

\section{New Example of Jawarneh Manifold}

Let us consider $\mathrm{R}^{4}$ endowed with the Riemannian metric [5],

3.1) $d^{2}=\mathrm{g}_{\mathrm{ij}} \mathrm{dx} \mathrm{x}^{\mathrm{i}} \mathrm{dx} \mathrm{x}^{\mathrm{j}}=\left(\mathrm{x}^{4}\right)^{\frac{4}{3}}\left[\left(\mathrm{dx}^{1}\right)^{2}+\left(\mathrm{dx}^{4}\right)^{2}\right]+\left(\mathrm{dx}^{2}\right)^{2}+\left(\mathrm{dx}^{3}\right)^{2}$,

where $i, j=1,2,3,4$. Then it is known [5] that the only non vanishing Ricci tensors and the curvature tensors are, 
$\Gamma_{14}^{1}=\Gamma_{44}^{4}=\frac{2}{3 x^{4}}=-\Gamma_{11}^{4}$

3.2) $R_{1441}=\frac{-2}{9\left(x^{4}\right)^{\frac{2}{3}}}$,

3.3) $S_{11}=S_{44}=\frac{-2}{3\left(\mathrm{x}^{4}\right)^{2}}$.

And the scalar curvature $\mathrm{r}=\frac{-4}{3\left(x^{4}\right)^{\frac{10}{3}}} \neq 0$.

Sincek $=\frac{1}{n-1}$ we can verify the definition by (1.1) byverifying only the following relation:

3.4) $R_{1441}=k\left[S_{44} g_{11}+S_{14} g_{41}\right]$.

Using (3.2), (3.3) and the value of a aboveon (3.4) we get,

$$
\begin{aligned}
\text { R.H.S. } & =\frac{1}{n-1}\left[S_{44} g_{11}+S_{14} g_{41}\right] \\
& =\frac{1}{3}\left[\frac{-2}{3\left(\mathrm{x}^{4}\right)^{2}}\left(\mathrm{x}^{4}\right)^{\frac{4}{3}}+0\right] \\
& =\frac{-2}{9\left(x^{4}\right)^{\frac{2}{3}}}=\text { L.H.S. }
\end{aligned}
$$

The other cases are trivially true. Hence $\mathrm{R}^{4}$ along with the metric $\mathrm{g}$ defined by (3.1) is Jawarneh manifold. Thus we can state,

Theorem3.1) A Riemannian manifold $\left(\mathrm{M}^{4}, \mathrm{~g}\right)$ endowed with the metric (3.1) is Jawarneh manifold with nonconstant scalar curvature.

\section{References}

[1] British Journal of Mathematics \& Computer Science, 7(3): 191-196, 2015

[2] K. Yano and M. Kon: Structures on Manifolds, World scientific 1989.

[3] K. Yano: On Semi-Symmetric Metric Connection. Rav. Roum. Math. Pures Appl. (Bucharest), 1970, 15(9), 1579-1586.

[4] B.B.Sinha: An introduction to Modern Differential Geometry, Kalyani Publishers, New Delhi, 1982.

[5] S. Kumar: On weakly W3-symmetric manifolds, Acta Univ. Palacki. Olomuc., Fac. rer. nat., Mathematica 50, 1 (2011) 53-71

[6] Narlikar JV.: General relativity and gravitation. The Macmillan Co. of India; 1978. 